\title{
BURNER-STABILIZED CELLULAR FLAMES
}

\author{
BY \\ DAVID O. OLAGUNJU (University of Delaware. Newark, Delaware) \\ AND \\ BERNARD J. MATKOWSKY (Northwestern University, Evanston, Illinois)
}

\begin{abstract}
We consider a model that governs the behavior of a premixed flame anchored on a flat burner. We show that a steady planar flame is stable for Lewis numbers $L$ in the interval $L_{l}<L<L_{u}$ where $L_{l}<1$ and $L_{u}>1$ and unstable otherwise.

We derive and analyze a Ginzburg-Landau equation for this problem in a neighborhood of the critical Lewis number $L_{l}$. We find that a family of spatially periodic solutions which correspond to stationary cellular flames, bifurcates from the basic solution. Subcritical bifurcations are found to be unstable. In the supercritical case there is a range of wavenumbers satisfying the Eckhaus criterion, within which these solutions are stable.

We also find spatially periodic solutions modulated by slowly varying amplitudes. The amplitude may be stationary spatially periodic, stationary solitary, or a travelling wave.

1. Introduction. In the diffusional-thermal theory of flame propagation, two types of instability are known to exist. These are the pulsating and cellular instabilities which occur when the Lewis number, defined as the ratio of thermal to mass diffusivities, is sufficiently bigger and smaller than unity, respectively.

Pulsating instabilities in combustion were studied in the theoretical works of Matkowsky and Sivashinsky [21], Matkowsky and Olagunju [16, 18, 19], Margolis and Matkowsky [13], and Margolis et al. [11], to mention just a few, and the experimental work of Blackshear et al., [2] and Mapp et al. [10].

Studies of cellular instabilities appear in the theoretical papers of Matkowsky, Putnick, and Sivashinsky [20], Sivashinsky [24, 25], Margolis and Sivashinsky [14], and Buckmaster [3] (detailed bibliographies can be found in Buckmaster and Ludford [4], Margolis and Matkowsky [12], and Sivashinsky [25]). Experimental observations of cellular flames have been reported and are well documented in the literature. Extensive references can be found in Markstein [15], Gaydon and Wolfhard [8], Lewis and von Elbe [9], and Buckmaster and Ludford [4].
\end{abstract}

Received June 1, 1989.

This research was supported in part by D. O. E. Grant DE-FG02-87ER25027.

(C)1990 Brown University 
In this paper we show that cellular flames arise as a bifurcation from a planar flame. In Buckmaster [3], a similar analysis was carried out using a different burner model. His single mode analysis was, however, restricted to long wavelength instabilities and to flames with large stand-off distances. In this analysis we place no restriction either on the wavelength or on the stand-off distance and instead of a single mode analysis we consider a continuous band of wave numbers centered around the most unstable wave number. Hence even for parameter values for which Buckmaster's analysis yields stable bifurcation, we find a range of wave numbers for which the bifurcated states are unstable.

2. The mathematical model. We consider a two-dimensional premixed flame anchored on a flat burner (Fig. 1). The model we use is a straightforward extension to two dimensions of the one used in Matkowsky and Olagunju [17] in which the burner is modelled as a heat sink. This approach was first used by Carrier, Fendell, and Bush [5]. We make the usual assumptions of weak thermal expansion of the gas, large activation energy and Lewis number close to unity. We also assume that reaction can be modelled by one-step Arrhenius kinetics; and for mathematical simplicity we take the temperature of the burner to be equal to that of the fresh mixture. We introduce the nondimensional parameter

$$
M=\frac{\widetilde{E}}{\widetilde{R} \widetilde{T}_{a}}\left(1-\frac{\widetilde{T}_{u}}{\widetilde{T}_{a}}\right) \gg 1,
$$

where $\widetilde{E}$ is the dimensional activation energy, $\widetilde{T}_{u}$ and $\widetilde{T}_{a}$ are the dimensional fresh mixture and adiabatic flame temperatures, respectively, and $\widetilde{R}$ is the gas constant.

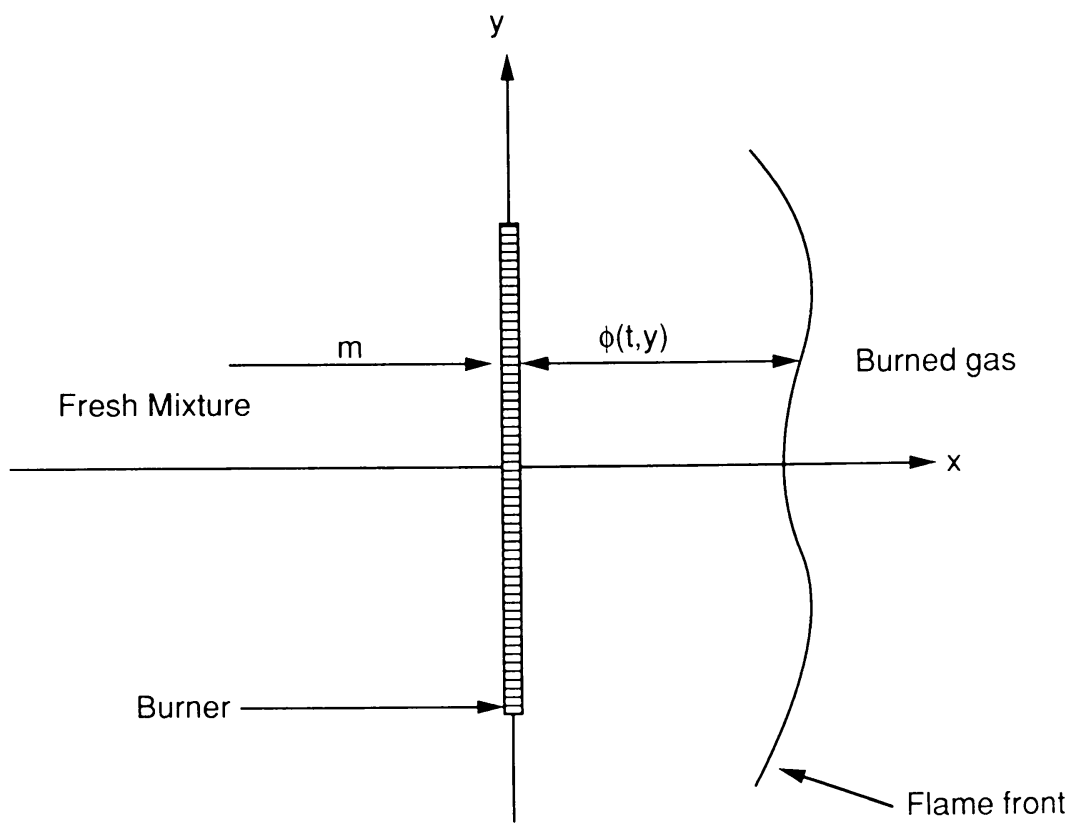

FIG. 1. A two-dimensional flame stabilized on a flat burner. 
We seek perturbation expansions of the nondimensional temperature $T\left(t, x_{1}, x_{2}\right)$ and mass fraction $Y\left(t, x_{1}, x_{2}\right)$ as

$$
\begin{gathered}
T=\frac{\widetilde{T}-\widetilde{T}_{u}}{\widetilde{T}_{a}-\widetilde{T}_{u}} \sim \Theta+\frac{T_{1}}{M}+\frac{T_{2}}{M^{2}}+\ldots, \\
Y=\frac{\widetilde{Y}_{\widetilde{Y}_{u}}}{\widetilde{Y}_{u}} \sim Y_{0}+\frac{Y_{1}}{M}+\frac{Y_{2}}{M^{2}}+\ldots,
\end{gathered}
$$

where $\tilde{Y}_{u}$ is the mass fraction of the fresh mixture. In addition, we scale the Lewis number $L$ and the heat loss coefficient $H$ as

$$
\begin{gathered}
L=1+\frac{\beta}{M}, \\
H=\frac{K}{M} .
\end{gathered}
$$

The boundary conditions $T_{\infty}=T\left(t,+\infty, x_{2}\right)$ and $Y\left(t,+\infty, x_{2}\right)$ are not known a-priori and have to be determined from the analysis, but we assume that $T_{\infty}-1=$ $O(1 / M)$. In the limit of large activation energy, the reaction zone becomes a thin sheet of flame whose location in nondimensional rectangular cartesian coordinates $\left(x_{1}, x_{2}\right)$ is given by

$$
x_{1}=\Phi\left(t, x_{2}\right),
$$

where $\Phi$ is to be determined. The burner is located at $x_{1}=0$. We assume that reaction comes to completion at the flame front so that $Y \equiv 0$ behind the flame. Introducing a coordinate system attached to the flame front

$$
x=x_{1}-\Phi\left(t, x_{2}\right), \quad y=x_{2},
$$

the model is given by

$$
\begin{gathered}
\Theta+Y_{0} \equiv 1 \\
\frac{\partial \Theta}{\partial t}+\left(m-\frac{\partial \Phi}{\partial t}\right) \frac{\partial \Theta}{\partial x}=\Delta \Theta+\left\{1+\left(\frac{\partial \Phi}{\partial y}\right)^{2}\right\}^{1 / 2} \exp \left(\frac{S}{2}\right) \delta(x), \\
\frac{\partial S}{\partial t}+\left(m-\frac{\partial \Phi}{\partial t}\right) \frac{\partial S}{\partial x}=\Delta S+\beta \Delta \Theta-K \Theta \delta(x+\Phi)
\end{gathered}
$$

subject to the boundary conditions

$$
\begin{gathered}
\Theta \equiv 1 \text { for } x>0, \quad \frac{\partial S}{\partial x} \rightarrow 0 \quad \text { as } x \rightarrow+\infty, \\
\Theta \rightarrow 0, \quad S \rightarrow 0 \text { as } x \rightarrow-\infty, \\
|\Theta|<\infty, \quad|S|<\infty \text { as } y \rightarrow \pm \infty .
\end{gathered}
$$

Here the variable $S$ is defined by

$$
S=T_{1}+Y_{1},
$$

$\delta$ is the Dirac delta function and the Laplacian in the moving coordinate system is

$$
\Delta=\frac{\partial^{2}}{\partial x^{2}}+\frac{\partial^{2}}{\partial y^{2}}-\frac{\partial^{2} \Phi}{\partial y^{2}} \frac{\partial}{\partial x}-2 \frac{\partial \Phi}{\partial y} \frac{\partial^{2}}{\partial x \partial y}+\left(\frac{\partial \Phi}{\partial y}\right)^{2} \frac{\partial^{2}}{\partial x^{2}} .
$$

After solving for $\Theta$ and $S$ and $\Phi$ in (2.9)-(2.13), we can determine $Y_{0}$ from (2.8). 


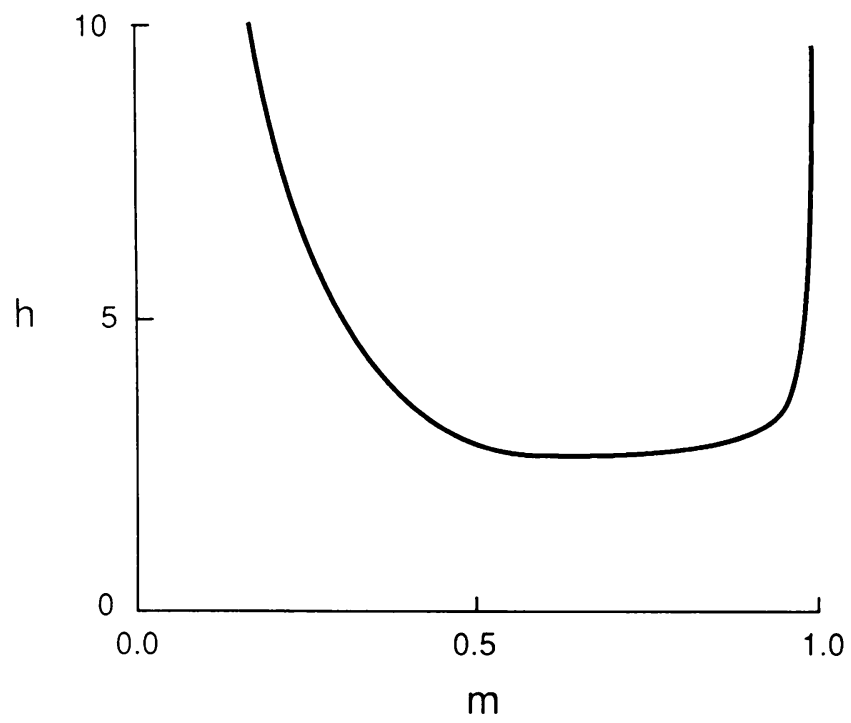

FIG. 2. Plot of $h$ vs. $m$ for $K=1$.

3. The basic solution and its linear stability. The problem (2.9)-(2.13) has a steady solution given by

$$
\begin{gathered}
\Theta_{0}(x)= \begin{cases}1, & x>0, \\
e^{m x}, & x<0,\end{cases} \\
S_{0}(x)= \begin{cases}B, & x>0, \\
B-\beta m x e^{m \cdot x}, & -h<x<0, \\
B e^{m(x+h)}-\beta m x e^{m x}, & x<-h,\end{cases} \\
\Phi_{0}=h,
\end{gathered}
$$

where

$$
B=2 \ln (m),
$$

and

$$
h=\frac{1}{m} \ln \left(\frac{-K}{B m}\right) \text {. }
$$

This solution, which we refer to as the basic solution, represents a stationary planar flame located at a stand-off distance $x_{1}=h$. Given the flow rate $m$ and heat loss parameter $K$, we determine $B$, which is the $O(1 / M)$ correction to the flame temperature, and the stand-off distance $h$ from (3.4) and (3.5), respectively. We find that $h$ is a $U$-shaped function of $m$ (see Fig. 2) but only the branch on which $h \rightarrow+\infty$ as $m \rightarrow 1^{-}$is of physical interest because as $m \rightarrow 0, B \rightarrow-\infty$ and the basic solution becomes unbounded. Therefore we restrict the value of $m$ so that it is bounded away from zero. For further discussion of the basic solution see Matkowsky and Olagunju [17]. 
We now consider the linear stability of the basic solution. To this end we introduce the perturbations

$$
\begin{gathered}
\phi=\Phi-h, \\
w=\Theta-\Theta_{0}(x)-\phi \frac{d \Theta_{0}}{d x}, \\
z=S-S_{0}(x)-\phi \frac{d S_{0}}{d x} .
\end{gathered}
$$

From (3.7) we obtain

$$
\phi=\frac{1}{m}[w]_{0} .
$$

The notation $[f]_{a}$ denotes the jump in $f$ across $x=a$

$$
[f]_{a}=f\left(t, a^{+}, y\right)-f\left(t, a^{-}, y\right) .
$$

Substituting (3.6)-(3.8) into (2.9)-(2.13) and linearizing about $\phi=w=z=0$ we obtain the linear problem

$$
\begin{gathered}
\frac{\partial w}{\partial t}+m \frac{\partial w}{\partial x}-\nabla^{2} w=0, \quad x \neq 0,-h, \\
\frac{\partial z}{\partial t}+m \frac{\partial z}{\partial x}-\nabla^{2} z-\beta \nabla^{2} w=0, \quad x \neq 0,-h,
\end{gathered}
$$

subject to the jump conditions

$$
\begin{gathered}
{[z]_{0}+\beta[w]_{0}=0,} \\
{\left[\frac{\partial w}{\partial x}\right]_{0}-m[w]_{0}+\frac{m}{2} z\left(t, 0^{+}, y\right)=0} \\
{\left[\frac{\partial z}{\partial x}\right]_{0}+\beta\left[\frac{\partial w}{\partial x}\right]_{0}+\beta m[w]_{0}=0} \\
{[z]_{-h}=0,} \\
{\left[\frac{\partial z}{\partial x}\right]_{-h}-K w(t,-h, y)=0}
\end{gathered}
$$

and the boundary conditions

$$
\begin{gathered}
w \equiv 0 \text { for } x>0, \quad \frac{\partial z}{\partial x} \rightarrow 0 \quad \text { as } x \rightarrow+\infty, \\
w \rightarrow 0, \quad z \rightarrow 0 \quad \text { as } x \rightarrow-\infty .
\end{gathered}
$$

This problem has solutions of the form

$$
\begin{aligned}
\left(\begin{array}{c}
w \\
z
\end{array}\right) & =R e^{\omega t+i k y}\left(\begin{array}{c}
W \\
Z
\end{array}\right)+\text { c.c. }, \\
W & = \begin{cases}0, & x>0, \\
-e^{p x}, & x<0,\end{cases}
\end{aligned}
$$

and

$$
Z= \begin{cases}\frac{2 l}{m} e^{l x}, & x>0, \\ C e^{l x}+D e^{p x}+\frac{\beta\left(p^{2}-k^{2}\right)}{p-l} x e^{p x}, & -h<x<0, \\ E e^{p x}+\frac{\beta\left(p^{2}-k^{2}\right)}{p-l} x e^{p x}, & x<-h,\end{cases}
$$


where $R$ is an arbitrary complex constant and c.c. denotes complex conjugate. The coefficients appearing in (3.20)-(3.21) are defined by

$$
\begin{gathered}
p=\frac{1}{2}\left\{m+\left[m^{2}+4 \omega+4 k^{2}\right]^{1 / 2}\right\}, \\
l=m-p, \\
C=\frac{2 l}{m}+\frac{\beta\left(l^{2}-k^{2}\right)}{(p-l)^{2}}, \\
D=\beta+\frac{2 l}{m}-C,
\end{gathered}
$$

and

$$
E=D+C e^{(p-l) h} .
$$

The solution (3.19) is nontrivial if and only if the following dispersion relation is satisfied

$$
2 l(l-p)^{2}+\beta m\left(l^{2}-k^{2}\right)+K m(l-p) e^{h(l-p)}=0 .
$$

The basic solution is stable (unstable) according as $\mathfrak{R}(\omega)<0 \quad(>0)$. Equation (3.27) possesses two neutral stability boundaries on which $\mathfrak{R}(\omega)=0$. On one $\omega \equiv 0$, while on the other only $\mathfrak{F}(\omega) \neq 0$. The former delineates the region corresponding to the cellular instability while the latter to that of the time-periodic (pulsating) instability. The stability diagram for $K=1$ and for selected values of $m$ is shown in Fig. 3. The picture does not change much with $K$. In this figure, the curve corresponding to $m=1$ is the same as that obtained by Sivashinsky [24] for adiabatic flames. For fixed values of $K$, the region of the pulsating instability increases as $m$ increases while that of the cellular instability decreases with $m$. As in the adiabatic case, the cellular instability is confined to the region $\beta<-2$. The cellular instability boundary is given explicitly by

$$
\beta=-2-\frac{8 k^{2}}{m^{2}}+\frac{2 K \alpha e^{-h \prime}}{m(m-\alpha)},
$$

where $\alpha^{2}=m^{2}+4 k^{2}$.

4. Nonlinear analysis. In this section we carry out a bifurcation analysis from the stationary planar flame (the basic solution) into a stationary cellular (nonplanar) flame. Hence, we confine our attention to the lower part of the stability diagram, i.e., the region $\beta<0$. For admissible values of the flow rate $m$, the neutral stability curve has a local maximum at $\left(\beta_{0}, k_{0}\right)$ with $k_{0} \neq 0$ (Fig. 3). We seek steady nonplanar solutions in a neighborhood of this point, using perturbation analysis. Thus we introduce a small parameter $\varepsilon$ to be defined below, scaled variables

$$
\tau=\varepsilon^{2} t, \quad \eta=\varepsilon y,
$$


and expand as

$$
\begin{gathered}
\beta \sim \beta_{0}\left(1+\nu \varepsilon^{2}\right)+\ldots, \\
\phi=\Phi-h \sim \varepsilon \phi_{1}+\varepsilon^{2} \phi_{2}+\ldots, \\
w=\Theta-\Theta_{0}-\phi \frac{d \Theta_{0}}{d x} \sim \varepsilon w_{1}+\varepsilon^{2} w_{2}+\ldots, \\
z=S-S_{0}-\phi \frac{d S_{0}^{0}}{d x} \sim \varepsilon z_{1}+\varepsilon^{2} z_{2}+\ldots,
\end{gathered}
$$

where the basic solution $S_{0}(x)$ is expanded as

$$
S_{0} \sim S_{0}^{0}+\varepsilon S_{0}^{1}+\varepsilon^{2} S_{0}^{2}+\ldots
$$

Substituting (4.1)-(4.6) into the nonlinear problem (2.9)-(2.13) and equating coefficients of like powers of $\varepsilon$, we obtain the following sequence of problems for the recursive determination of $w_{j}, z_{j}, \phi_{j}$ for $j=1,2,3, \ldots$

$$
\begin{gathered}
\frac{\partial w_{j}}{\partial t}+m \frac{\partial w_{j}}{\partial x}-\nabla^{2} w_{j}=r_{j 1}, \quad x \neq 0,-h, \\
\frac{\partial z_{j}}{\partial t}+m \frac{\partial z_{j}}{\partial x}-\nabla^{2}\left(z_{j}+\beta_{0} w_{j}\right)=r_{j 2}, \quad x \neq 0,-h,
\end{gathered}
$$

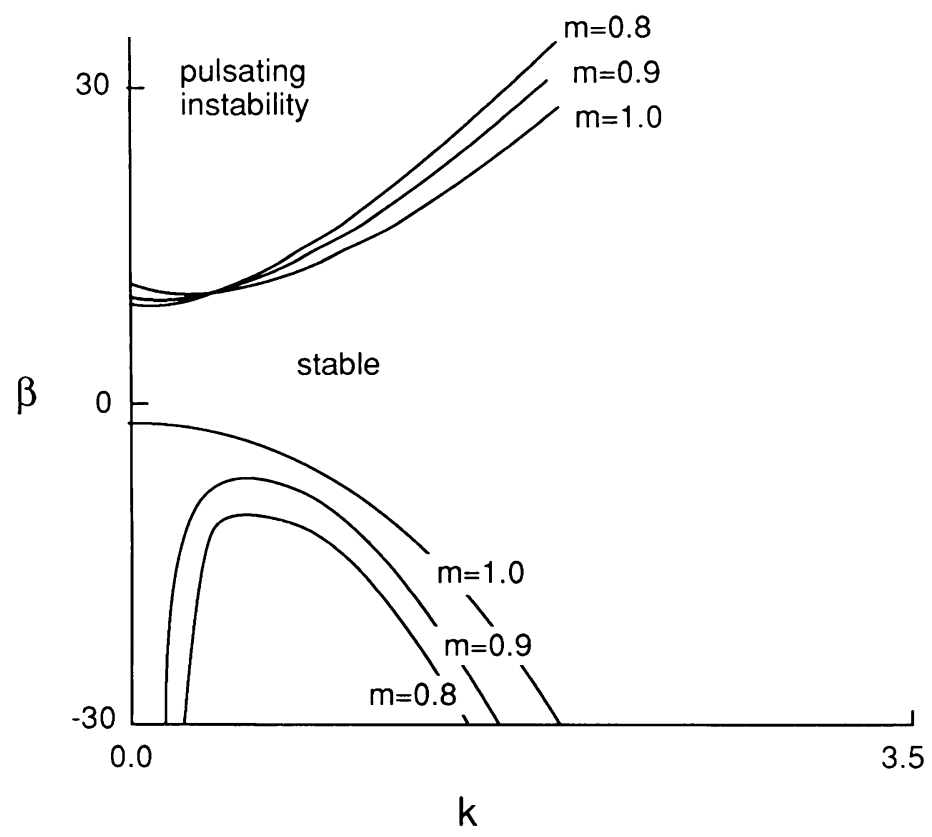

FIG. 3. Stability diagram for $K=1$. 
subject to the jump conditions

$$
\begin{gathered}
\phi_{j}=\frac{1}{m}\left[w_{j}\right]_{0}, \\
{\left[z_{j}\right]_{0}+\beta_{0}\left[w_{j}\right]_{0}=0,} \\
{\left[\frac{\partial w_{j}}{\partial x}\right]_{0}-m\left[w_{j}\right]_{0}+\frac{m}{2} z_{j}\left(\tau, 0^{+}, y\right)=\rho_{j 1},} \\
{\left[\frac{\partial z_{j}}{\partial x}\right]_{0}+\beta_{0}\left[\frac{\partial w_{j}}{\partial x}\right]_{0}+\beta_{0} m\left[w_{j}\right]_{0}=\rho_{j 2},} \\
{\left[z_{j}\right]_{-h}=\rho_{j 3},} \\
{\left[\frac{\partial z_{j}}{\partial x}\right]_{-h}-K w_{j}(\tau,-h, y)=\rho_{j 4}}
\end{gathered}
$$

and boundary conditions

$$
\begin{gathered}
w_{j} \equiv 0 \text { for } x>0, \quad \frac{\partial z_{j}}{\partial x} \rightarrow 0 \quad \text { as } x \rightarrow+\infty, \\
w_{j} \rightarrow 0, \quad z_{j} \rightarrow 0 \quad \text { as } x \rightarrow-\infty .
\end{gathered}
$$

The small parameter $\varepsilon$ is defined by

$$
\left\langle\left(\begin{array}{c}
w \\
z
\end{array}\right),\left(\begin{array}{c}
w^{*} \\
z^{*}
\end{array}\right)\right\rangle_{1}=\varepsilon
$$

The vector $\left(w^{*}, z^{*}\right)^{T}$ is the long-time solution of the adjoint problem given in Appendix $\mathrm{B}$ where the scalar product is also defined. This definition of $\varepsilon$ implies that

$$
\left\langle\left(\begin{array}{c}
w_{j} \\
z_{j}
\end{array}\right),\left(\begin{array}{c}
w^{*} \\
z^{*}
\end{array}\right)\right\rangle_{1}=\delta_{j 1}
$$

where $\delta_{j 1}$ is the Kronecker delta. The inhomogeneous terms $r_{j 1}, r_{j 2}, \rho_{j 1}, \rho_{j 2}$, $\rho_{j 3}$, and $\rho_{j 4}$ for $j=1-3$ are given in Appendix A. For $j=1$ these quantities are all zero, and the general long-time solution of the homogeneous problem (4.7)-(4.16) is given by

$$
\left(\begin{array}{c}
w_{1} \\
z_{1} \\
\phi_{1}
\end{array}\right)=\left(R e^{i k_{0} y}+\bar{R} e^{-i k_{0} y}\right)\left(\begin{array}{c}
W(x) \\
Z(x) \\
1 / m
\end{array}\right),
$$

where $W$ and $Z$ are given by (3.20) and (3.21), respectively, but with $\beta, k$, and $\omega$ replaced by $\beta_{0}, k_{0}$, and zero, respectively. All other modes corresponding to $k \neq k_{0}$ decay in time. The complex coefficient $R(\tau, \eta)$, whose conjugate is denoted $\bar{R}$, must be determined.

The problems (4.7)-(4.16) with $j \geq 2$ are inhomogeneous forms of the problem with $j=1$ and are in general not solvable unless certain solvability conditions are satisfied. These conditions are derived in Appendix B.

The solvability conditions are identically satisfied for the problem with $j=2$ and the solution is given by

$$
\left(\begin{array}{c}
w_{2} \\
z_{2}
\end{array}\right)=\left(R^{2} e^{2 i k_{0} y}+\bar{R}^{2} e^{-2 i k_{0} y}\right)\left(\begin{array}{c}
W_{22}(x) \\
Z_{22}(x)
\end{array}\right)+R \bar{R}\left(\begin{array}{c}
F(x) \\
G(x)
\end{array}\right),
$$

where the functions $W_{22}, Z_{22}, F(x)$, and $G(x)$ are given in Appendix C. 


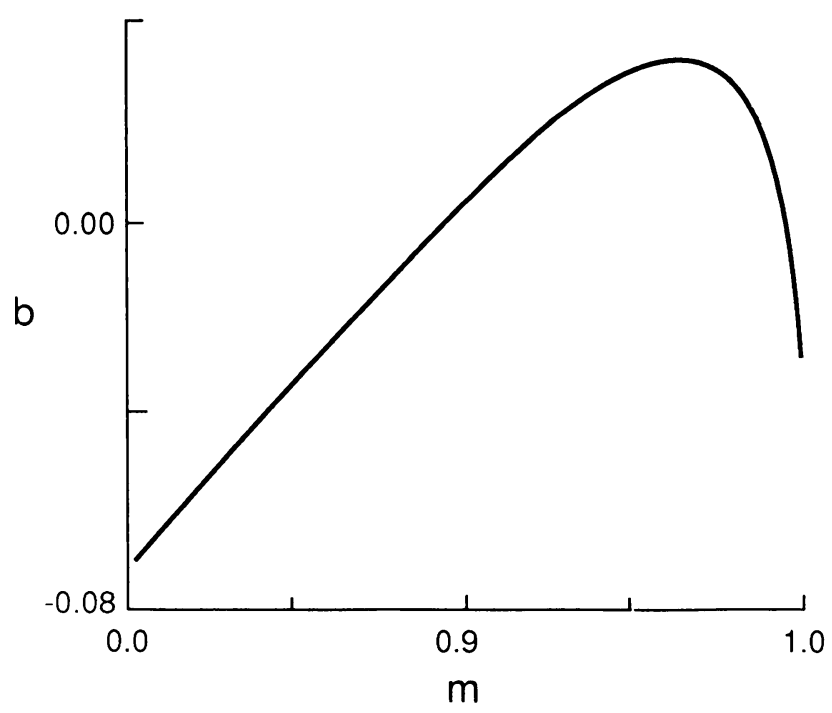

FIG. 4a. Plot of $b$ vs. $m$ for $K=1$.

Applying the solvability conditions for $j=3$, we obtain the evolution equation

$$
\frac{\partial R}{\partial \tau}=\nu a R+d \frac{\partial^{2} R}{\partial \eta^{2}}+b|R|^{2} R
$$

The real coefficients $a, b$, and $d$ depend on the parameters $m$ and $K$. For all values of $K$ considered, $a$ and $d$ are positive if $m \in(0,1)$. For $m$ close to $1, b$ is negative but as $m$ decreases $b$ becomes positive and then turns negative again (Fig. $4 a)$. When $b=0,(4.21)$ is degenerate and additional terms are required to describe the evolution of $R$. Therefore we shall consider only the case $b \neq 0$. Equation (4.21), referred to as the Ginzburg-Landau equation, has been derived by several authors, e.g., in fluid flow problems [22, 23] and by Cohen et al. [6] for nonlinear diffusion processes.

5. Analysis of the amplitude equation. In this section we analyze the amplitude equation (4.21). The basic solution is given by $R=0$. We first seek nontrivial stationary solutions of the form $R=\rho(\eta) e^{i \theta(\eta)}$. When $\rho$ is a constant we obtain stationary spatially periodic solutions, otherwise we obtain stationary modulated spatially periodic solutions. Substituting for $R$ in (4.21) we obtain the pair of equations

$$
\begin{gathered}
d \rho^{\prime \prime}+\nu a \rho-d \rho\left(\theta^{\prime}\right)^{2}+b \rho^{3}=0, \\
\left(\rho^{2} \theta^{\prime}\right)^{\prime}=0,
\end{gathered}
$$

where primes denote differentiation with respect to $\eta$.

5.1. Steady periodic solutions. Here we seek solutions of (5.1) and (5.2) of the form $\rho=\rho_{0}$, where $\rho_{0}$ is a constant. It then follows that $\theta=\kappa \eta$ where $\kappa$ is a constant. Thus

$$
\rho_{0}^{2}=\frac{d \kappa^{2}-\nu a}{b}
$$


provided

$$
\frac{d \kappa^{2}-\nu a}{b} \geq 0
$$

In the subcritical case $(\nu<0),(5.3)$ is satisfied if $b>0$ while in the supercritical case $(\nu>0)$ it is satisfied only by a range of wave numbers $\kappa$ given by $\kappa^{2} \geq(\leq)$ $\nu a / d$ according as $b>(<) 0$.

In order to determine the stability of the stationary periodic cellular flame $(5.3)$, we consider a perturbation $\chi$ given by

$$
R=\rho_{0} e^{i \kappa \eta}+\chi(\tau, \eta) e^{i \kappa \eta}
$$

Substituting (5.5) into (4.21) and linearizing we obtain

$$
\frac{\partial \chi}{\partial \tau}=\left(\nu a-d \kappa^{2}+2 b \rho_{0}^{2}\right) \chi+d\left(\frac{\partial^{2} \chi}{\partial \eta^{2}}+2 i \kappa \frac{\partial \chi}{\partial \eta}\right)+b \rho_{0}^{2} \bar{\chi}
$$

If we now set $\chi=u+i v$, then $u$ and $v$ satisfy

$$
\begin{gathered}
\frac{\partial u}{\partial \tau}=2 b \rho_{0}^{2} u-2 \kappa \frac{\partial v}{\partial \eta}+d \frac{\partial^{2} u}{\partial \eta^{2}} \\
\frac{\partial v}{\partial \tau}=2 \kappa \frac{\partial u}{\partial \eta}+d \frac{\partial^{2} v}{\partial y^{2}}
\end{gathered}
$$

Equations (5.7)-(5.8) have nontrivial solutions of the form

$$
\left(\begin{array}{l}
u \\
v
\end{array}\right)=e^{\Omega \tau+i \mu \eta}\left(\begin{array}{l}
\Gamma_{1} \\
\Gamma_{2}
\end{array}\right)
$$

provided $\Omega$ satisfies

$$
\left(\Omega+\mu^{2} d\right)\left(\Omega+\mu^{2} d-2 b \rho_{0}^{2}\right)-(2 \kappa \mu)^{2}=0 .
$$

The solution (5.3) is stable (unstable) acording as $\Omega<0 \quad(>0)$. The condition for stability is satisfied if and only if $b<0$ and

$$
\kappa^{2}<\nu a /(3 d) \text {. }
$$

This last condition, usually referred to as the Eckhaus criterion [7], requires that $\nu>0$, since as remarked earlier $a$ and $d$ are positive. Hence, only supercritical bifurcations may be stable. Furthermore, (5.11) implies that a mode with wave number $k$ is stable if and only if

$$
\frac{k_{-}-k_{0}}{\sqrt{3}}<k-k_{0}<\frac{k_{+}-k_{0}}{\sqrt{3}} .
$$

Here $k_{-}$and $k_{+}$are the points of intersection of the neutral stability curve and the line $\beta=\beta_{c}$ (see Fig. 3), where $\beta_{c}$ is a constant less than $\beta_{0}$. Therefore the wave number $k$ of a stable stationary cellular flame must be restricted to the interval $(5.12)$. 
5.2. Periodic and solitary envelopes. We now seek more general stationary solutions of (5.1) and (5.2). From (5.2) we have $\left(\rho^{2} \theta^{\prime}\right)=h$ where $h$ is an arbitrary constant. Equation (5.11) then becomes

$$
\rho^{\prime \prime}+\frac{\nu a}{d} \rho-\frac{h^{2}}{\rho^{3}}+\frac{b}{d} \rho^{3}=0
$$

which after one integration yields

$$
\left(\rho^{\prime}\right)^{2}+\frac{\nu a}{d} \rho^{2}+\frac{h^{2}}{\rho^{2}}+\frac{b}{2 d} \rho^{4}=E,
$$

where $E$ is an arbitrary constant of integration. As shown in [1, 22, 23], if $\nu a / d>0$ and $b / d<0$, the above equation possesses periodic envelopes as well as solitary envelopes. Since $a$ and $d$ are always positive these conditions reduce to $\nu>0$ and $b<0$. The solution corresponding to the periodic envelope can be written in terms of elliptic functions.

We consider in more detail the case $h=0$, which implies that $\theta$ is constant. In the supercritical case $(\nu>0)$ a phase plane analysis of (5.13) shows that if $b>0$, the origin is the only singular point and it is a center. On the other hand if $b<0$, there are three singular points, one at the origin (center) and the others at $\pm \sqrt{\nu a / d}$ (saddle points). Of these only the nonnegative solutions are admissible. The phase plane analysis shows that the only bounded solutions are the equilibrium points $\rho=0$ which corresponds to the basic solution, and $\rho=\sqrt{\nu a / d}$ which corresponds to the spatially periodic solution obtained above, so that the analysis reduces to that considered in the previous section.

In the subcritical case $(\nu<0)$ there is no bounded solution if $b<0$. For $b>0$ there are three singular points, the same ones as above. However, the origin is now a saddle while the other two are centers. The centers correspond to periodic solutions which are given by elliptic functions and the separatrix is a solution of solitary type.

5.3. Travelling waves. Here we seek travelling wave solutions of (4.21). Let $\zeta=\eta-c \tau$, so that travelling wave solutions satisfy

$$
d R^{\prime \prime}+c R^{\prime}+\nu a R+b R^{3}=0,
$$

where $c$ and $R$ are real and primes denote differentiation with respect to $\zeta$. Equation (5.15) can be written as the first-order system

$$
\begin{gathered}
R^{\prime}=S, \\
S^{\prime}=-\frac{c}{d} S-\frac{\nu a}{d} R-b R^{3} .
\end{gathered}
$$

The above system has three critical points at $(R, S)=(0,0)$ and $\left( \pm R_{0}, 0\right)$ where $R_{0}^{2}=-\nu a / b>0$. Consider the supercritical case $\nu>0$. The points $\left( \pm R_{0}, 0\right)$ are spiral points while the origin is a spiral if $0<|c|<\sqrt{4 \nu a d}$ and an improper node if $\sqrt{4 \nu a d}<|c|$. As shown by Cohen et al. [6] the only bounded solutions are the separatrices joining the origin to the other two critical points. These solutions correspond to cellular flames modulated by slowly varying travelling waves. Since the 


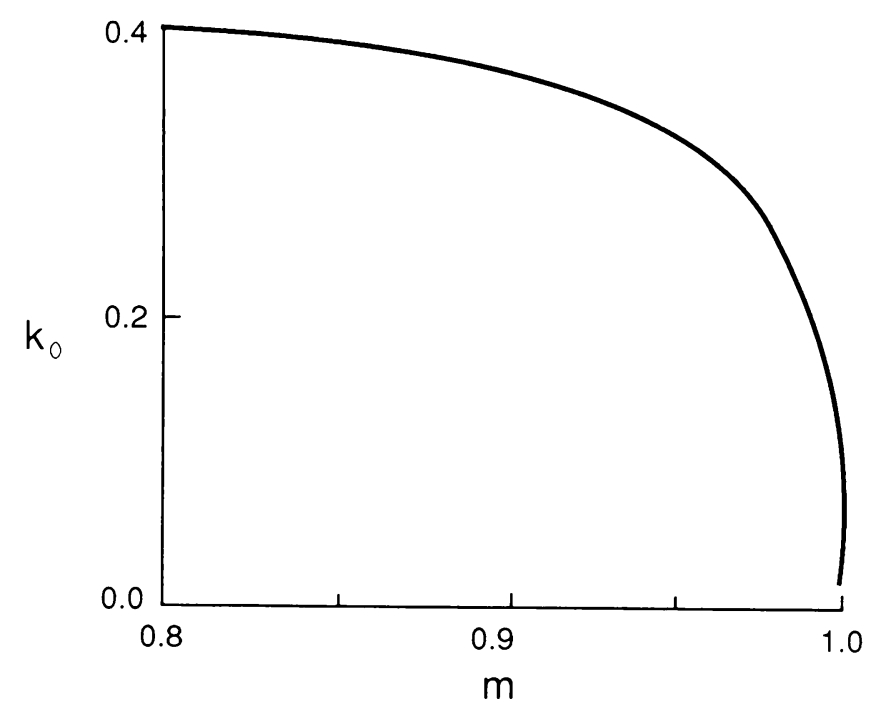

Fig. 4b. Plot of $k_{0}$ vs. $m$ for $K=1$.

origin is a spiral, a solution starting close to the origin and lying on the separatrix is initially oscillatory in time. The amplitudes of these oscillations grow and approach the value $R_{0}$ asymptotically. This phenomenon, referred to as a precursor wave, has been observed experimentally in some chemical reactors [cf. 6]. In flame theory, it describes a mechanism for transition from a stationary planar flame to a stationary cellular flame. When the stationary planar flame loses stability, a cellular flame with very small amplitude begins to develop. Its amplitude exhibits growing oscillations in time, eventually saturating at the value $R_{0}$, thus yielding a stationary cellular flame.

6. Summary and conclusions. We have shown that when the reduced Lewis number $\beta$ is decreased below the critical value $\beta_{0}$, a nonplanar cellular flame bifurcates from the planar flame. Since $\beta_{0}<0$ it follows that the bifurcation point corresponds to a value of the Lewis number $L_{l}<1$.

The shape of the cellular flame front is given by

$$
\Phi(y) \sim h+\frac{2 \rho}{m} \varepsilon \cos (k y)+\varepsilon^{2} \rho^{2}\left\{2 j_{22} \cos (2 k y)+j_{23}\right\}+\ldots,
$$

where $j_{22}=\frac{1}{m}\left[W_{22}\right]_{0}$ and $j_{23}=\frac{1}{m}[F]_{0}$ are computed from the expressions in Appendix $\mathrm{C}$ and $\rho_{0}$ is given by (5.3). The wave number of a cell is $k=k_{0}+\varepsilon \kappa+o(\varepsilon)$ where $\kappa$ is given by $(5.11)$. As shown in Fig. $4 \mathrm{~b}, k_{0}$ decreases monotonically to zero as $m$ increases to unity. Lewis and von Elbe [9] have reported that cell size increases with burning velocity as this analysis shows. From (6.1) we obtain the mean stand-off distance of the cellular flame as

$$
\bar{\Phi}=h+\varepsilon^{2} j_{23} \rho^{2}+o\left(\varepsilon^{2}\right)
$$

The quantity $j_{23}$ is always negative (see Fig. 4c) so that the mean stand-off distance of the cellular flame is less than that of the planar flame. The temperature at the flame 


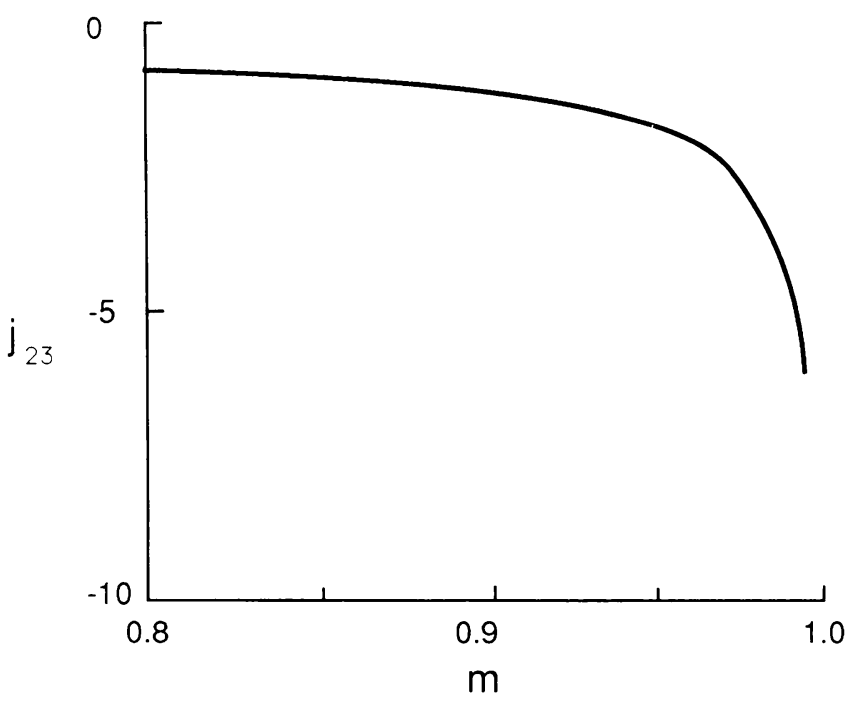

FiG. 4c. Plot of $j_{23}$ vs. $m$ for $K=1$.

front is given by

$$
\begin{aligned}
T_{f}=1 & +\frac{B}{M}+\frac{4 l}{m} \varepsilon \rho \cos (k y) \\
& +\frac{\rho^{2}}{M} \varepsilon^{2}\left[2\left(\frac{2 l^{2}}{m^{2}}+B_{22}\right) \cos (2 k y)+\left(\frac{4 l^{2}}{m^{2}}+B_{23}\right)\right]+o\left(\varepsilon^{2}\right) .
\end{aligned}
$$

The parameter $M$ is the modified activation energy defined in $(2.1)$ and $(1+B / M)$ is the temperature at the front of the planar flame. The constants $B_{22}$ and $B_{23}$ which appear above are defined in Appendix C. From Fig. 5 we see that the cellular flame is hottest at the troughs and coldest at the crests in agreement with experimental results.

As shown in the previous section, when $b<0$ bifurcation is subcritical $c_{1}$ id unstable. For $b>0$ bifurcation is supercritical. However, the range of wave numl ins for which the bifurcated state is stable is limited, according to the Eckhaus criterion, to the band (5.12) about the critical wave number $k_{0}$. From Fig. $4 a$ we see that as $m$ increases, $b$ changes sign twice from negative to positive and then to negative. Let $m_{1}$ and $m_{2}$ be the values of $m$ at which $b$ changes sign with $0<m_{1}<$ $m_{2}$. Suppose further that $\left(m_{0}, 1\right)$ is the range of admissible values of $m$ where $m_{0}<m_{1}$. Then for flow rates $m \in\left(m_{0}, m_{1}\right)$ or $m \in\left(m_{2}, 1\right), b<0$ and so as the Lewis number $L$ is decreased past the critical value $L_{l}$, the stationary planar flame loses stability and there is a bifurcation to a stable stationary cellular flame. In his analysis, Buckmaster [3] considered flames with large stand-off distances and looked for bifurcation to spatially periodic solutions with wave number equal to $k_{0}$. When the flow rate $m$ is close to 1 , we obtain the large stand-off limit he considered (see Fig. 2). Unlike Buckmaster, however, we consider an entire band of wave numbers centered around $k_{0}$. Our analysis reduces to his in the appropriate limit. We also found solutions which the single-mode analysis cannot describe. These 


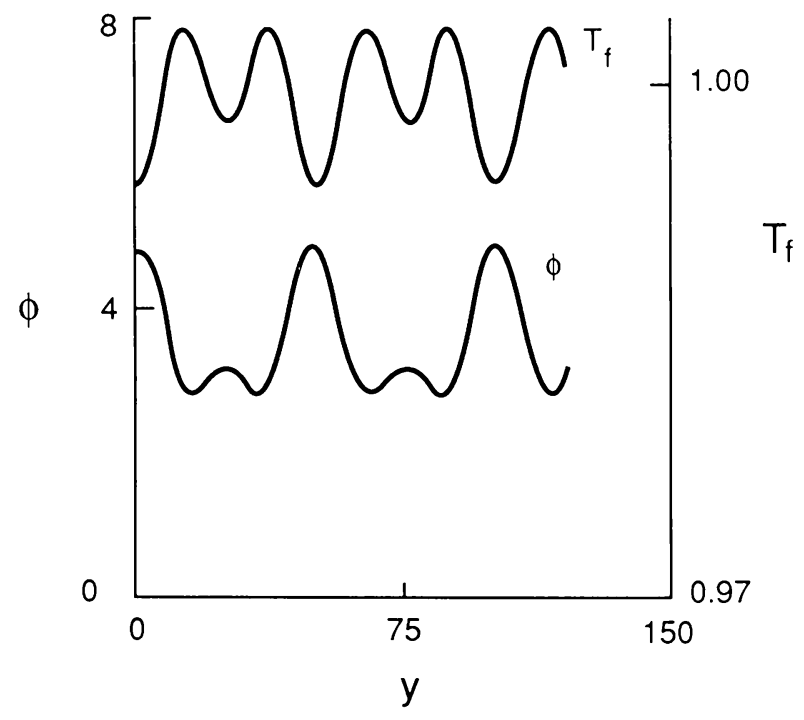

Fig. 5. Plots of cellular flame front $\Phi$ and flame temperature $T_{f}$ for $K=1, m=.999$ and $\varepsilon=0.5$.

include stationary spatially-periodic solutions modulated by slowly varying periodic or solitary envelopes. If $m \in\left(m_{1}, m_{2}\right)$, so that $b>0$, the cellular flame is unstable. In this interval, we showed that there are other solutions corresponding to stationary cellular flames modulated by slowly varying stationary periodic or solitary envelopes or by stationary slowly varying travelling wave envelopes.

A. Appendix A. The inhomogeneous terms $r_{j 1}, r_{j 2}, \rho_{j 1}, \rho_{j 2}, \rho_{j 3}$, and $\rho_{j 4}$ appearing in (4.7)-(4.14) for $j=1-3$, are

$$
\begin{gathered}
r_{11}=r_{12}=\rho_{11}=\rho_{12}=\rho_{13}=\rho_{14}=0, \\
r_{21}=-\frac{\partial^{2} \phi_{1}}{\partial y^{2}}\left(\frac{\partial w_{1}}{\partial x}+\phi_{1} \frac{\partial^{2} \Theta_{0}}{d x^{2}}\right)-\left(\frac{d \phi_{1}}{d y}\right)^{2} \frac{d^{2} \Theta_{0}}{d x^{2}}-2 \frac{\partial \phi_{1}}{\partial y} \frac{\partial^{2} w_{1}}{\partial x \partial y}, \\
r_{22}=-\frac{\partial^{2} \phi_{1}}{\partial y^{2}}\left[\frac{\partial z_{1}}{\partial x}+\beta_{0} \frac{\partial w_{1}}{\partial x}+\phi_{1}\left(\frac{d^{2} S_{0}^{0}}{d x^{2}}+\beta_{0} \frac{d^{2} \Theta_{0}}{d x^{2}}\right)\right] \\
-\left(\frac{\partial \phi_{1}}{\partial y}\right)^{2}\left(\frac{d^{2} S_{0}^{0}}{d x^{2}}+\beta_{0} \frac{d^{2} \Theta_{0}}{d x^{2}}\right)-2 \frac{\partial \phi_{1}}{\partial y}\left(\frac{\partial^{2} z_{1}}{\partial x \partial y}+\beta_{0} \frac{\partial^{2} w_{1}}{\partial x \partial y}\right), \\
\rho_{21}=\frac{m}{2}\left(\frac{\partial \phi_{1}}{\partial y}\right)^{2}-\frac{m}{2}\left\{z_{1}\left(\tau, 0^{+}, y\right)\right\}^{2}, \\
\rho_{23}=\frac{\phi_{1}^{2}}{2}\left[\frac{d^{2} S_{0}^{0}}{d x^{2}}\right]_{-h}+\phi_{1}\left[\frac{\partial z_{1}}{\partial x}\right]_{-h},
\end{gathered}
$$




$$
\begin{aligned}
& \rho_{24}=\frac{\phi_{1}^{2}}{2}\left[\frac{d^{3} S_{0}^{0}}{d x^{3}}\right]_{-h}+\phi_{1}\left[\frac{d^{2} z}{d x^{2}}\right]_{-h}-\left(\frac{\partial \phi_{1}}{\partial y}\right)^{2}\left[\frac{d S_{0}^{0}}{d x}\right]_{-h} \\
& -\left.K\left(\phi_{1} \frac{\partial w_{1}}{\partial x}+\frac{\phi_{1}^{2}}{2} \frac{d^{2} \Theta_{0}}{d x^{2}}\right)\right|_{x=-h} \\
& r_{31}=-\frac{\partial w_{1}}{\partial \tau}-\frac{\partial^{2} \phi_{1}}{\partial y^{2}}\left(\frac{\partial w_{2}}{\partial x}+\phi_{2} \frac{d^{2} \Theta_{0}}{d x^{2}}\right)-\frac{\partial^{2} \phi_{2}}{\partial y^{2}}\left(\frac{\partial w_{1}}{\partial x}+\phi_{1} \frac{d^{2} \Theta_{0}}{d x^{2}}\right) \\
& -2 \frac{\partial \phi_{1}}{\partial y} \frac{\partial \phi_{2}}{\partial y} \frac{d^{2} \Theta_{0}}{d x^{2}}-2 \frac{\partial \phi_{1}}{\partial y} \frac{\partial^{2} w_{2}}{\partial x \partial y}-2 \frac{\partial \phi_{2}}{\partial y} \frac{\partial^{2} w_{1}}{\partial x \partial y} \\
& +\left(\frac{\partial \phi_{1}}{\partial y}\right)^{2}\left(\frac{\partial w_{1}}{\partial x}+\phi_{1} \frac{d^{2} \Theta_{0}}{d x^{2}}\right) \\
& r_{32}=-\frac{\partial z_{1}}{\partial \tau}-\frac{\partial^{2} \phi_{1}}{\partial y^{2}} \frac{d S_{0}^{2}}{d x}+\beta_{0} \nu \phi_{1} \frac{d^{3} \Theta_{0}}{d x^{3}}+\beta_{0} \nu \nabla^{2} w_{1} \\
& -\frac{\partial^{2} \phi_{1}}{\partial y^{2}}\left(\frac{\partial z_{2}}{\partial x}+\beta_{0} \frac{\partial w_{2}}{\partial x}+m \phi_{2} \frac{d S_{0}^{0}}{d x}\right)-2 m \frac{\partial \phi_{1}}{\partial y} \frac{\partial \phi_{2}}{\partial y} \frac{d S_{0}^{0}}{d x} \\
& -\frac{\partial^{2} \phi_{2}}{\partial y^{2}}\left(\frac{\partial z_{1}}{\partial x}+\beta_{0} \frac{\partial w_{1}}{\partial x}+m \phi_{1} \frac{d S_{0}^{0}}{d x}\right) \\
& -2 \frac{\partial \phi_{1}}{\partial y}\left(\frac{\partial^{2} z_{2}}{\partial x \partial y}+\beta_{0} \frac{\partial^{2} w_{2}}{\partial x \partial y}\right)-2 \frac{\partial \phi_{2}}{\partial y}\left(\frac{\partial^{2} z_{1}}{\partial x \partial y}+\beta_{0} \frac{\partial^{2} w_{1}}{\partial x \partial y}\right) \\
& +\left(\frac{\partial \phi_{1}}{\partial y}\right)^{2}\left(\frac{\partial^{2} z_{1}}{\partial x^{2}}+\beta_{0} \frac{\partial^{2} w_{1}}{\partial x^{2}}+m \phi_{1} \frac{d^{2} S_{0}^{0}}{d x^{2}}\right)-2 m \frac{\partial \phi_{1}}{\partial y} \frac{\partial \phi_{2}}{\partial y} \frac{d S_{0}^{0}}{d x}, \\
& \rho_{31}=-\left.\frac{m}{4}\left(z_{1} z_{2}\right)\right|_{x=0^{+}}+m \frac{\partial \phi_{1}}{\partial y} \frac{\partial \phi_{2}}{\partial y}-\frac{m}{48}\left(\left.z_{1}\right|_{x=0^{+}}\right)^{3} \\
& +\frac{m}{4}\left(\frac{\partial \phi_{1}}{\partial y}\right)^{2}\left(\left.z_{1}\right|_{x=0^{+}}\right) \\
& \rho_{32}=-\beta_{0} \nu\left(\left[\frac{\partial w_{1}}{\partial x}\right]_{0}-m\left[w_{1}\right]_{0}\right) \\
& \rho_{33}=\phi_{1} \phi_{2}\left[\frac{d^{2} S_{0}^{0}}{d x^{2}}\right]_{-h}+\phi_{1}\left[\frac{\partial z_{2}}{\partial x}\right]_{-h}+\phi_{2}\left[\frac{\partial z_{1}}{\partial x}\right]_{-h} \\
& -\frac{1}{2} \phi_{1}^{2}\left[\frac{\partial^{2} z_{1}}{\partial x^{2}}\right]_{-h}-\frac{1}{2} \phi_{1}^{3}\left[\frac{d^{3} S_{0}^{0}}{d x^{3}}\right]_{-h} \text {, }
\end{aligned}
$$




$$
\begin{aligned}
\rho_{34}= & \phi_{2}\left[\frac{\partial^{2} z_{1}}{\partial x^{2}}\right]_{-h}+\phi_{1}\left[\frac{\partial^{2} z_{2}}{\partial x^{2}}\right]_{-h}+\phi_{1} \phi_{2}\left[\frac{d^{3} S_{0}^{0}}{d x^{3}}\right]_{-h} \\
& -\frac{1}{2} \phi_{1}^{2}\left[\frac{\partial^{3} z_{1}}{\partial x^{3}}\right]_{-h}-\frac{1}{2} \phi_{1}^{3}\left[\frac{d^{4} S_{0}^{0}}{d x^{4}}\right]_{-h} \\
& -2 \frac{\partial \phi_{1}}{\partial y} \frac{\partial \phi_{2}}{\partial y}\left[\frac{d S_{0}^{0}}{d x}\right]_{-h}-\left(\frac{\partial \phi_{1}}{\partial y}\right)^{2}\left[\frac{\partial z_{1}}{\partial x}\right]_{-h} \\
& +K\left(-\phi_{1} \frac{\partial w_{2}}{\partial x}-\phi_{2} \frac{\partial w_{1}}{\partial x}\right. \\
& \left.\quad-\phi_{1} \phi_{2} \frac{d^{2} \Theta_{0}}{d x^{2}}+\frac{1}{2} \phi_{1}^{2} \frac{\partial^{2} w_{1}}{\partial x^{2}}+\frac{1}{2} \phi_{1}^{3} \frac{d^{3} \Theta_{0}}{d x^{3}}\right)\left.\right|_{x=-h}
\end{aligned}
$$

B. Appendix B. The problem adjoint to the linear homogeneous problem defined in $(4.7)-(4.16)$ is given by

$$
\begin{gathered}
\frac{\partial w^{*}}{\partial t}+m \frac{\partial w^{*}}{\partial x}+\nabla^{2} w^{*}+\beta_{0} \nabla^{2} z^{*}=0, \quad x \neq 0,-h, \\
\frac{\partial z^{*}}{\partial t}+m \frac{\partial z^{*}}{\partial x}+\nabla^{2} z^{*}=0, \quad x \neq 0,-h,
\end{gathered}
$$

together with the jump conditions

$$
\begin{gathered}
{\left[z^{*}\right]_{0}=0,} \\
\frac{\partial w^{*}}{\partial x} \rightarrow 0 \text { as } x \rightarrow 0^{-} \\
{\left[\frac{\partial z^{*}}{\partial x}\right]_{0}+\left.\frac{m}{2} w^{*}\right|_{x=0^{-}}=0,} \\
{\left[z^{*}\right]_{-h}=\left[w^{*}\right]_{-h}=0,} \\
{\left[\frac{\partial z^{*}}{\partial x}\right]_{-h}=0,} \\
{\left[\frac{\partial w^{*}}{\partial x}\right]_{-h}-\left.K z^{*}\right|_{x=-h}=0}
\end{gathered}
$$

and the boundary conditions

$$
w^{*} \rightarrow 0, \quad z^{*} \rightarrow 0 \quad \text { as } x \rightarrow \pm \infty
$$

The inhomogeneous problem (4.7)-(4.17) is solvable if and only if the following condition is satisfied

$$
\begin{aligned}
\left\langle\left(\begin{array}{l}
r_{j 1} \\
r_{j 2}
\end{array}\right),\left(\begin{array}{c}
w^{*} \\
z^{*}
\end{array}\right)\right\rangle_{1}= & \left\langle\left(\begin{array}{c}
\rho_{j 1} \\
\rho_{j 2}
\end{array}\right),\left(\begin{array}{c}
\left.w^{*}\right|_{x=0^{-}} \\
\left.z^{*}\right|_{x=0^{+}}
\end{array}\right)\right\rangle_{2} \\
& +\left\langle\left(\begin{array}{c}
\rho_{j 3} \\
\rho_{j 4}
\end{array}\right),\left.\left(\begin{array}{c}
-m z^{*}-\frac{\partial z^{*}}{\partial x} \\
z^{*}
\end{array}\right)\right|_{x=-h^{+}}\right\rangle_{2},
\end{aligned}
$$


where the inner products are defined as

$$
\begin{gathered}
\left\langle\left(\begin{array}{l}
f_{1} \\
f_{2}
\end{array}\right),\left(\begin{array}{l}
g_{1} \\
g_{2}
\end{array}\right)\right\rangle_{1} \equiv \lim _{T \rightarrow+\infty} \frac{1}{T} \int_{0}^{T} \int_{0}^{2 \pi / k_{0}} \int_{-\infty}^{+\infty}\left(f_{1} g_{1}+f_{2} g_{2}\right) d t d y d x, \\
\left\langle\left(\begin{array}{l}
f_{1} \\
f_{2}
\end{array}\right),\left(\begin{array}{l}
g_{1} \\
g_{2}
\end{array}\right)\right\rangle_{2} \equiv \lim _{T \rightarrow+\infty} \frac{1}{T} \int_{0}^{T} \int_{0}^{2 \pi / k_{0}}\left(f_{1} g_{1}+f_{2} g_{2}\right) d t d y .
\end{gathered}
$$

The long-time solutions of the adjoint problem, $w^{*}, z^{*}$ are given in complex form as

$$
\left(\begin{array}{c}
w^{*} \\
z^{*}
\end{array}\right)=e^{ \pm i k_{0} y}\left(\begin{array}{c}
W^{*} \\
Z^{*}
\end{array}\right),
$$

where

$$
\begin{gathered}
W^{*}= \begin{cases}C^{*} e^{-\rho x}+\beta_{0} \frac{p^{2}-k_{0}^{2}}{p-l} x e^{-p x}, & x>0, \\
D^{*} e^{-l x}+E^{*} e^{-p x}+\beta_{0} \frac{l^{2}-k_{0}^{2}}{1-p} x e^{-l x}, & -h<x<0, \\
F^{*} e^{-l x}+\beta_{0} \frac{l^{2}-k_{0}^{2}}{l-p} x e^{-l x}, & x<-h,\end{cases} \\
Z^{*}= \begin{cases}e^{-p x}, & x>0, \\
e^{-l x}, & x<0 .\end{cases}
\end{gathered}
$$

The coefficients above are given by

$$
\begin{aligned}
& C^{*}=2(p-l) / m, \\
& E^{*}=-\left\{\frac{2 l}{m}+\beta_{0} \frac{l^{2}-k_{0}^{2}}{(p-l)^{2}}\right\}, \\
& D^{*}=C^{*}-E^{*}, \\
& F^{*}=D^{*}+E^{*} e^{(p-l) h},
\end{aligned}
$$

and $l$ and $p$ are given by (3.22) and (3.23) with $\omega=0$ and $k=k_{0}$.

C. Appendix C. The functions $W_{22}(x), Z_{22}(x), F(x)$, and $G(x)$ which appear in (4.20) are defined as

$$
\begin{gathered}
W_{22}(x)=\frac{1}{m} \frac{d W}{d x}+\frac{1}{2 m} \frac{d \Theta_{0}}{d x}+ \begin{cases}0, & x<0, \\
A_{22} e^{\lambda x}, & x<0,\end{cases} \\
Z_{22}(x)=\frac{1}{m} \frac{d Z}{d x}+\frac{1}{2 m^{2}} \frac{d^{2} S_{0}^{0}}{d x^{2}} \\
+\left\{\begin{array}{lc}
B_{22} e^{(m-\lambda) x}, & x>0, \\
C_{22} e^{(m-\lambda) x}+D_{22} e^{\lambda x}+\beta_{0} A_{22} \frac{\lambda^{2}-4 k_{0}^{2}}{m-2 \lambda} x e^{\lambda x}, & -h<x<0, \\
E_{22} e^{\lambda x}+\beta_{0} A_{22} \frac{\lambda^{2}-4 k_{0}^{2}}{m-2 \lambda} x e^{\lambda x}, & x<-h,
\end{array}\right.
\end{gathered}
$$

where

$$
\lambda=\frac{1}{2}\left\{m+\left(m^{2}+16 k_{0}^{2}\right)^{1 / 2}\right\}
$$


and the real coefficients $A_{22}, B_{22}, C_{22}, D_{22}$, and $E_{22}$ are the solutions of the linear equation

$$
\mathscr{M}\left(\begin{array}{l}
A_{22} \\
B_{22} \\
C_{22} \\
D_{22} \\
E_{22}
\end{array}\right)=\left(\begin{array}{c}
s_{1} \\
s_{2} \\
s_{3} \\
s_{4} \\
s_{5}
\end{array}\right)
$$

The matrix $\mathscr{M}$ is given by

$$
\mathscr{M}=\left(\begin{array}{ccccc}
-\beta_{0} & 1 & -1 & -1 & 0 \\
m-\lambda & m / 2 & 0 & 0 & 0 \\
-\beta_{0}\left(m+\lambda+\frac{\lambda^{2}-4 k_{0}^{2}}{m-2 \lambda}\right) & m-\lambda & \lambda-m & -\lambda & 0 \\
0 & 0 & e^{h(\lambda-m)} & e^{-h \lambda} & e^{-h \lambda} \\
-K e^{-h \lambda} & 0 & (m-\lambda) e^{h(\lambda-m)} & \lambda e^{-h \lambda} & -\lambda e^{-h \lambda}
\end{array}\right)
$$

while the right-hand side of (C.4) is

$$
\begin{gathered}
s_{1}=-\frac{2 l^{2}}{m^{2}}+\alpha_{22}+\frac{l C}{m}-\frac{\beta_{0}}{2}-\frac{\beta_{0} p}{m}, \\
s_{2}=-\frac{3 l^{2}}{2 m}-\frac{k_{0}^{2}}{2 m}-\frac{p^{2}}{m}+p, \\
s_{3}=-\frac{2 l^{3}}{m^{2}}+p \alpha_{22}+\delta_{22}+\frac{l^{2} C}{m}-\frac{\beta_{0} m}{2}-\frac{\beta_{0} p^{2}}{m}-\beta_{0} p, \\
s_{4}=\left\{\frac{p}{m}(D-E)-\alpha_{22}+\gamma_{22}\right\} e^{-p h}, \\
s_{5}=\left\{\frac{p^{2}}{m}(D-E)-p\left(\alpha_{22}-\gamma_{22}\right)\right\} e^{-p h}-\frac{B k_{0}^{2}}{m},
\end{gathered}
$$

and

$$
\begin{gathered}
\alpha_{22}=\frac{1}{m}\left(p D+\beta_{0} \frac{p^{2}-k_{0}^{2}}{p-l}\right), \\
\gamma_{22}=\frac{1}{m}\left(p E+\beta_{0} \frac{p^{2}-k_{0}^{2}}{p-l}\right), \\
\delta_{22}=\beta_{0} p \frac{p^{2}-k_{0}^{2}}{m(p-l)} .
\end{gathered}
$$

The functions $F(x)$ and $G(x)$ are given by

$$
\begin{aligned}
F(x)=\frac{A_{23}}{m} \frac{d \Theta_{0}}{d x}+\frac{2}{m} \frac{d W}{d x}, \\
G(x)=\frac{2}{m} \frac{d Z}{d x} \\
+ \begin{cases}B_{23}, & x>0, \\
C_{23}+D_{23} e^{m x}-\beta_{0} A_{23} m x e^{m x}, & -h<x<0, \\
E_{23} e^{m x}-\beta_{0} A_{23} m x e^{m x}, & x<-h,\end{cases}
\end{aligned}
$$


where the coefficients $A_{23}, B_{23}, C_{23}, D_{23}$, and $E_{23}$ are the solutions of the linear equation

$$
\mathscr{N}\left(\begin{array}{l}
A_{23} \\
B_{23} \\
C_{23} \\
D_{23} \\
E_{23}
\end{array}\right)=\left(\begin{array}{l}
t_{1} \\
t_{2} \\
t_{3} \\
t_{4} \\
t_{5}
\end{array}\right) \text {. }
$$

The matrix $\mathcal{N}$ is defined by

$$
\mathscr{N}=\left(\begin{array}{ccccc}
-\beta_{0} & 1 & -1 & -1 & 0 \\
0 & 1 & 0 & 0 & 0 \\
-\beta_{0} m & 0 & 0 & -m & 0 \\
0 & 0 & 1 & e^{-m h} & -e^{-m h} \\
-K e^{-m h} & 0 & 0 & m e^{-m h} & -m e^{-m h}
\end{array}\right)
$$

while the right-hand side of $(\mathrm{C} .16)$ is

$$
\begin{gathered}
t_{1}=-\frac{4 l^{2}}{m^{2}}+\frac{2 l C}{m}+2 \alpha_{22}-2 \frac{\beta_{0} p}{m}, \\
t_{2}=\frac{2}{m}\left(-\frac{2 p^{2}}{m}+2 p-\frac{3 l^{2}}{m}+\frac{k_{0}^{2}}{m}\right), \\
t_{3}=-\frac{4 l^{3}}{m^{2}}+\frac{2 l^{2} C}{m}+2 p \alpha_{22}+2 \delta_{22}-\frac{2 \beta_{0} p^{2}}{m}-2 \beta_{0} p, \\
t_{4}=\frac{2 p}{m}(D-E) e^{-p h}-B+2\left(\alpha_{22}-\gamma_{22}\right) e^{-p h}, \\
t_{5}=\frac{2 p^{2}}{m}(D-E) e^{-p h}-B m+2 k_{0}^{2} \frac{B}{m}-K e^{-m h} \\
+2 p\left(\alpha_{22}-\gamma_{22}\right) e^{-p h} .
\end{gathered}
$$

\section{REFERENCES}

[1] D. J. Benney and A. C. Newell, The propagation of nonlinear wave envelopes, J. Math. Phys. 46 , 133-139 (1967)

[2] J. I. Blackshear, J. W. Mapp, and M. Gorman, An experimental study of pulsating low pressure flames, Comb. Sc. Tech. 35, 311-315 (1984)

[3] J. D. Buckmaster, Polyhedral flames-an exercise in bimodal bifurcation analysis, SIAM J. Appl. Math. 44, 40-55 (1984)

[4] J. D. Buckmaster and S. S. Ludford, Lectures on Mathematical Combustion, CBMS Regional Conference Series in Applied Mathematics 43 (1983), SIAM. Philadelphia

[5] G. F. Carrier, F. E. Fendell, and W. B. Bush, Stoichiometry and flameholder effects on a onedimensional flame, Comb. Sc. Tech. 18, 33-46 (1978)

[6] D. S. Cohen, F. C. Hoppennsteadt. and R. M. Miura, Slow/y modulated oscillations in nonlinear diffiusion processes, SIAM J. Appl. Math. 33, 217-229 (1977)

[7] W. Eckhaus, Studies in Nonlinear Stability. Theory, Springer-Verlag, New York. 1965

[8] A. A. Gaydon and H. G. Wolfhard, Flames, Chapman and Hall, London, 1978

[9] B. Lewis and G. von Elbe. Combustion. Flames and Explosion of Gases, Academic Press. New York, 1987

[10] J. W. Mapp, J. I. Blackshear. and M. Gorman. Nonsteady, nonplanar modes of propagation in premixed burner-stabilized flames, Comb. Sc. Tech. 43, 217-225 (1985) 
[11] S. B. Margolis, H. G. Kaper, G. K. Leaf, and B. J. Matkowsky, Bifurcation of pulsating and spinning reaction fronts in condensed two-phase combustion, Comb. Sc. Tech. 43, 127-165 (1985)

[12] S. B. Margolis and B. J. Matkowsky, Nonlinear stability and bifurcation in transition from laminar to turbulent flame propagation, Comb. Sc. Tech. 34, 45-77 (1983)

[13] S. B. Margolis and B. J. Matkowsky, Flame propagation in channels: secondary bifurcation to quasi-periodic pulsations, SIAM J. Appl. Math. 45, 93-129 (1985)

[14] S. B. Margolis and G. I. Sivashinsky, Flame propagation in vertical channels: bifurcation to bimodal cellular flames, SIAM J. Appl. Math. 44, 344-368 (1984)

[15] G. H. Markstein, Nonsteady Flame Propagation, Pergamon Press, Oxford, 1964

[16] B. J. Matkowsky and D. O. Olagunju, Propagation of a pulsating reaction front in a gaseous combustible mixture, SIAM J. Appl. Math. 39. 290-300 (1980)

[17] B. J. Matkowsky and D. O. Olagunju, Pulsations in a burner-stabilized premixed plane flame, SIAM J. Appl. Math. 40, 551-562 (1981)

[18] B. J. Matkowsky and D. O. Olagunju, Travelling waves along the front of a pulsating flame, SIAM J. Appl. Math. 42, 486-501 (1982)

[19] B. J. Matkowsky and D. O. Olagunju, Spinning wares in gaseous combustion. SIAM J. Appl. Math. 42, 1138-1156 (1982)

[20] B. J. Matkowsky, L. J. Putnick, and G. I. Sivashinsky, A nonlinear theory of cellular flames, SIAM J. Appl. Math. 38, 489-504 (1980)

[21] B. J. Matkowsky and G. I. Sivashinsky, Propagation of a pulsating reaction front in solid fuel combustion, SIAM J. Appl. Math. 35, 465-478 (1978)

[22] A. C. Newell and J. A. Whitehead, Finite bandwidth, finite amplitude convection, J. Fluid Mech. 38, 279-303 (1969)

[23] L. A. Segel, Distant side-walls cause slow amplitude modulation of cellular convection. J. Fluid. Mech. 38, 203-224 (1969)

[?4] G. I. Sivashinsky, Diffiusional thermal theory of cellular flames, Comb. Sc. Tech. 15, 137 (1977)

[25] G. I. Sivashinsky, Instabilities, pattern formation, and turbulence in flames, Annual Review of Fluid Mechanics 15, 179-199 (1983) 